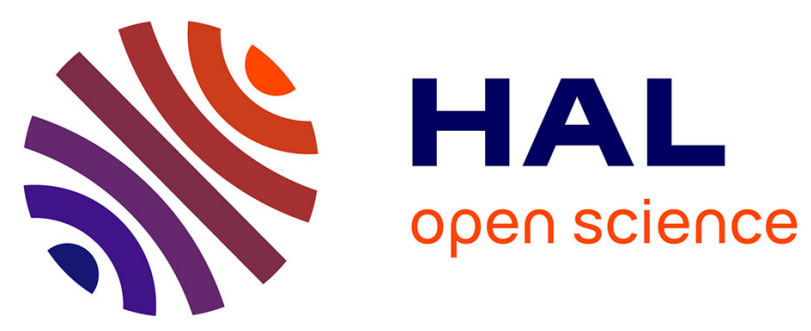

\title{
Modélisation multi-échelle du comportement électrique de nano-composites $\mathrm{Cu}-\mathrm{Nb}$
}

Tang Gu, Eveline Hervé-Luanco, Henry Proudhon, L. Thilly, J.-B. Dubois, Florence Lecouturier, Olivier Castelnau, Samuel Forest

\section{- To cite this version:}

Tang Gu, Eveline Hervé-Luanco, Henry Proudhon, L. Thilly, J.-B. Dubois, et al.. Modélisation multiéchelle du comportement électrique de nano-composites $\mathrm{Cu}-\mathrm{Nb}$. Matériaux \& Techniques, 2015, 103 (3), pp.9. 10.1051/mattech/2015033 . hal-02145858

\section{HAL Id: hal-02145858 https://hal.science/hal-02145858}

Submitted on 3 Jun 2019

HAL is a multi-disciplinary open access archive for the deposit and dissemination of scientific research documents, whether they are published or not. The documents may come from teaching and research institutions in France or abroad, or from public or private research centers.
L'archive ouverte pluridisciplinaire HAL, est destinée au dépôt et à la diffusion de documents scientifiques de niveau recherche, publiés ou non, émanant des établissements d'enseignement et de recherche français ou étrangers, des laboratoires publics ou privés. 


\title{
Modélisation multi-échelle du comportement électrique de nano-composites $\mathrm{Cu}-\mathrm{Nb}$
}

\author{
T. Gu ${ }^{1,2}$, E. Herve-Luanco ${ }^{2}$, H. Proudhon ${ }^{2}$, L. Thilly ${ }^{3}$, J.-B. Dubois ${ }^{3}$, \\ F. Lecouturier ${ }^{4}$, O. Castelnau ${ }^{1}$ et S. Forest ${ }^{2}$
}

\begin{abstract}
Résumé - Les fils composites nanostructurés et architecturés cuivre-niobium, qui sont de bons candidats pour la génération de champs magnétiques intenses, allient une limite d'élasticité élevée et une excellente conductivité électrique. Ils sont élaborés par co-déformation d'un assemblage composite $\mathrm{Cu}-\mathrm{Nb}$. La microstructure, multi-échelle, est formée de $85^{3}$ motifs élémentaires de $\mathrm{Cu}-\mathrm{Nb}$ de taille caractéristique nanométrique. Afin d'étudier le lien entre la conductivité électrique effective et la microstructure, deux méthodes d'homogénéisation sont appliquées : l'une, en champs moyens (modèle auto-cohérent généralisé), dans laquelle une microstructure formée de motifs co-cylindriques répartis aléatoirement est considérée, et l'autre, en champs complets (éléments finis), dans laquelle l'aspect périodique de la microstructure expérimentale est pris en compte. Les effets de la taille des constituants élémentaires (nm), de la température, ainsi que de la densité de dislocations, sur la conductivité locale sont considérés. Le caractère multi-échelle du matériau est pris en compte grâce à un processus itératif. Les conductivités effectives longitudinale et transversale obtenues avec les deux méthodes sont en excellent accord, montrant un moindre effet de la distribution des fibres sur ces propriétés. Ces résultats reproduisent également les données expérimentales disponibles.
\end{abstract}

Mots clés : Composites architecturés / matériaux polycristallins / méthodes d'homogénéisation / modélisation multi-échelle / conductivité électrique

\begin{abstract}
Multiscale modeling of the electrical behavior of $\mathrm{Cu}-\mathrm{Nb}$ nanocomposites. Nanostructured and architectured copper niobium composite wires are very good candidates for the generation of very high magnetic fields as they combine both high strength and high electrical conductivity. They are fabricated by co-deformation of a $\mathrm{Cu}-\mathrm{Nb}$ composite assembly. A multiscale microstructure formed by $85^{3} \mathrm{Cu}-\mathrm{Nb}$ elementary patterns with dimensions of the order of nanometers, is obtained. In order to investigate the link between the effective electrical conductivity and the wire microstructure, two homogenization methods are applied: first, using a mean field theory (generalized self-consistent model) in which a microstructure formed by co-cylindrical long fibers with a random distribution is considered, and second a full field method (Finite Elements) in which the periodic character of the experimental microstructure is taken into account. The size effect of components (nm), temperature, and dislocation density are taken into account into the definition of the local conductivity. The multiscale character of the material is taken into consideration through an iterative process. The longitudinal and transverse effective conductivities obtained by both methods perfectly match with each other, showing the limited influence of the effect of fiber distribution for this behavior. Results also compare well with the available experimental data.
\end{abstract}

Key words: Multiscale composites / polycrystalline materials / homogenization methods / multiscale modeling / electrical conductivity

\footnotetext{
1 Laboratoire PIMM, CNRS UMR 8006, Arts \& Métiers ParisTech, 151 Bd de l'hopital, 75013 Paris, France Tang.GU@ensam.eu

2 Centre des matériaux, CNRS UMR 7633, Mines ParisTech, BP 87, 91003 Evry Cedex, France

3 Institut Pprime, UPR 3346, CNRS, University of Poitiers, ISAE-ENSMA, SP2MI, Boulevard Marie et Pierre Curie, BP 30179, 86962 Futuroscope Chasseneuil Cedex, France

${ }^{4}$ Laboratoire National des Champs Magnétiques Intenses, UPR 3228 CNRS-UPS-INSA-UJF, 143 avenue de Rangueil, 31400 Toulouse, France
} 


\section{Introduction}

Les travaux présentés ici concernent l'étude de fils conducteurs composites nanostructurés à base de cuivreniobium $(\mathrm{Cu}-\mathrm{Nb})$, qui sont de bons candidats pour la réalisation de bobines permettant la génération des champs magnétiques très intenses $(>90 \mathrm{~T})$. Ces champs magnétiques sont devenus un outil expérimental et industriel essentiel [1-3]. Pour les produire, les fils conducteurs constituant les bobines doivent allier une limite d'élasticité et une conductivité électrique élevées [4]. Dans [5], un conducteur présentant une contrainte maximale de $1.9 \mathrm{GPa}$ à $77 \mathrm{~K}$ a été obtenu, pour une conductivité de $1.72 \mu \Omega^{-1} \mathrm{~cm}^{-1}$.

Les conducteurs nano-composites continus $\mathrm{Cu}-\mathrm{Nb}$ sont élaborés par un procédé de déformation plastique sévère, basé sur des cycles successifs d'extrusion à chaud, d'étirage à froid et d'empilements [4]. Comme illustré dans la figure 1 , ce procédé d'élaboration conduit à la formation d'une structure multi-échelle complexe de la matrice de cuivre et la nanostructuration des renforts de niobium. Il en résulte une nanostructuration du matériau composite, qui a un effet important sur les propriétés du conducteur, lui permettant d'atteindre les propriétés recherchées.

La figure 1 présente la microstructure multi-échelle des fils $\mathrm{Cu}-\mathrm{Nb}$ (microstructure appelée "co-cylindriques" dans [7]) : à l'échelle du Zoom 1, les nanotubes de niobium (Nb-t) sont séparés par de fins canaux de cuivre $(\mathrm{Cu}-0)$, et ils contiennent un filament de cuivre $(\mathrm{Cu}-\mathrm{f})$. On définit l'échelle effective du Zoom 1 par l'assemblage de 85 de ces motifs élémentaires. Sur le Zoom 2, des canaux de $\mathrm{Cu}(\mathrm{Cu}-1)$ entourent 85 motifs élémentaires décrits dans le Zoom 1. Au niveau du Zoom 3, le fil est constitué de $85^{3}$ motifs de $\mathrm{Cu}-\mathrm{f} / \mathrm{Nb}-\mathrm{t} / \mathrm{Cu}-0$ de taille caractéristique nanométrique, et séparés par les canaux de cuivre $\mathrm{Cu}-1, \mathrm{Cu}-2$. Le Zoom 4 correspond à la microstructure du Zoom 3 entourée d'un cylindre de $\mathrm{Cu}-3$, reproduisant ainsi la structure expérimentale de la dernière étape d'élaboration (échelle macroscopique). En raison du processus d'élaboration itératif, tous les nanotubes de $\mathrm{Nb}$ ont subi la même déformation, et présentent donc des microstructures et des tailles caractéristiques similaires. Concernant le $\mathrm{Cu}$, les régions $\mathrm{Cu}-\mathrm{f}$ et $\mathrm{Cu}-0$ sont introduites lors de la toute première étape d'élaboration, alors que $\mathrm{Cu}-1, \mathrm{Cu}-2$, et $\mathrm{Cu}-3$ sont introduits successivement; les microstructures et les tailles caractéristiques de ces constituants sont donc différentes (voir le Tab. 1).

Les études réalisées précédemment sur ces matériaux concernent le procédé d'élaboration [8, 9], la caractérisation des matériaux $[10,11]$, la stabilité thermique [12], la modélisation atomistique des interfaces [13] et les propriétés mécaniques $[14,15]$. En outre, la conductivité électrique a été étudiée expérimentalement et théoriquement par Thilly [5] et Dubois [7] dans la direction longitudinale, i.e. selon l'axe du fil.

Dans ce travail, nous présentons une modélisation multi-échelle de la conductivité électrique effective du fil, en tenant compte de son aspect anisotrope. Le modèle Auto-Cohérent Généralisé (ACG, Sect. 2.1), initialement développé pour prédire le comportement élastique effectif [16-18], puis modifié par Joannes et Hervé-Luanco [19] pour les phénomènes de diffusion dans les composites à fibres longues $n$-phases isotropes transverses, est appliqué ici au cas de la conductivité électrique. Ce modèle considère une répartition aléatoire des motifs élémentaires. Un second modèle, basé sur la méthode des Eléments Finis et prenant en compte le caractère périodique de la microstructure expérimentale (HP dans Sect. 2.2), mais plus lourd au niveau numérique, est également appliqué [20-22]. Dans [5], les effets de la taille des constituants (nm), de la température, et de la densité de dislocations sur la conductivité locale sont pris en compte à l'aide de la loi de Dingle (Sect. 3.1). Les résultats théoriques et numériques obtenus, dans lesquels le caractère multi-échelle du matériau est pris en compte grâce à un processus itératif (Sect. 3.2), sont comparés à des mesures expérimentales (Sect. 4).

\section{Modélisation de la conductivité électrique effective des composites}

\subsection{Modèle auto-cohérent généralisé}

La figure 1 présente la disposition des fils de nanocomposite $\mathrm{Cu}-\mathrm{Nb}$ à différents échelles. Sur le Zoom 1, les fils co-cylindriques apparaissent avec trois couches : $\mathrm{Cu}-\mathrm{f} / \mathrm{Nb}-$ t/Cu-0. Le modèle Auto-Cohérent Généralisé (ACG) [19] considère qu'ils sont distribués aléatoirement. Ce schéma est développé de la manière suivante : d'abord le problème élémentaire d'une inclusion constituée de $n$ phases cocylindriques dans une matrice homogène, défini sur la figure 2, est résolu; puis la matrice est remplacée par le Milieu Homogène Équivalent (MHE), dont la conductivité effective $\underset{\widetilde{\boldsymbol{\sigma}}}{\widetilde{\alpha}}$ sera déterminée par une condition d'autocohérence (Fig. 3a).

\subsubsection{Problème cylindrique $n$-phase}

Nous commençons par présenter le problème élémentaire d'une inclusion dans une matrice homogène (problème cylindrique $n$-phase). Une inclusion cylindrique de $n$ couches est noyée dans une matrice infinie (phase $n+1$ ), chaque phase étant supposée homogène et isotrope transverse (l'axe de symétrie est ici confondu avec la direction longitudinale des fibres longues). Des systèmes de coordonnées cartésiennes $\left(x_{1}, x_{2}, x_{3}\right)$ et cylindriques $(r, \theta, z)$, de même origine, sont utilisés dans cette étude. L'axe $z$ est ici identique à l'axe $x_{1}$, et il est aligné avec la direction longitudinale des fibres longues (Fig. 2).

Comme dans Hervé et Zaoui [18], sur la figure 2, la phase $i$ se situe dans la couronne limitée par les deux cylindres concentriques de rayon $R_{i-1}$ et $R_{i}(i \in[1, n+$ $1]$ avec $\left.R_{0}=0, R_{n+1} \rightarrow \infty\right)$. Dans la phase $i, \sigma_{L}^{(i)}$ et $\sigma_{T}^{(i)}$ sont respectivement les conductivités longitudinale et 


\section{Zoom 3}

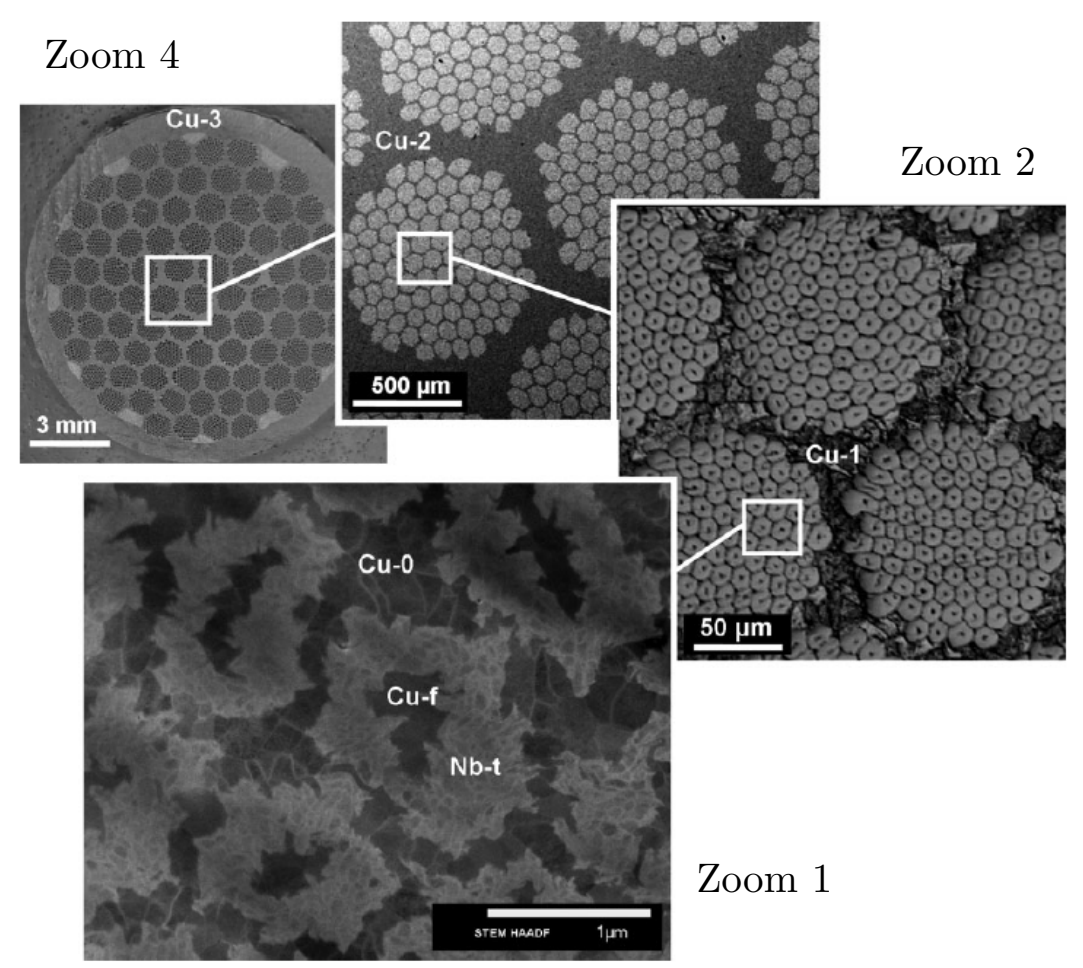

Fig. 1. Microstructure multi-échelle des conducteurs nano-composites Cu-Nb. Zoom 4, 3 et 2 : observations par Microscopie Électronique à Balayage (MEB), Zoom 1 : observation par Microscopie Électronique en Transmission (MET) [6,7].

Fig. 1. Multi-scale microstructure of $\mathrm{Cu}-\mathrm{Nb}$ nano-composite conductor. Zoom 4, 3 and 2: micrographs obtained by Scanning Electron Microscopy (SEM), Zoom 1: obtained by Transmission Electron Microscopy (TEM) [6, 7].

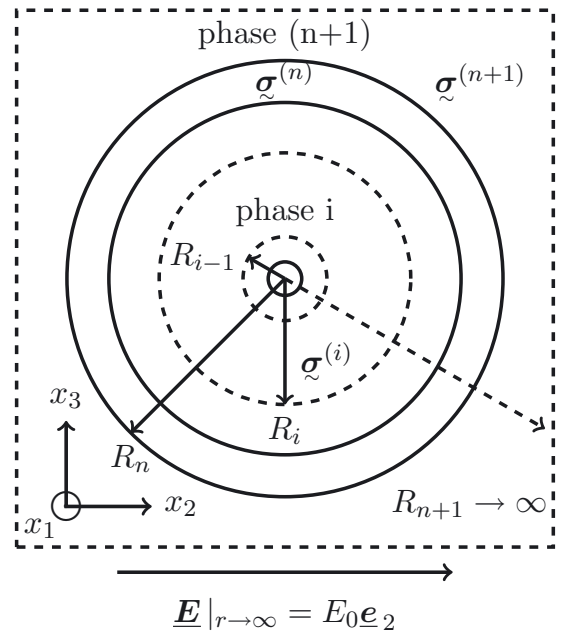

Fig. 2. Problème cylindrique $n$-phase (conduction électrique transversale).

Fig. 2. n-layered cylindrical problem (transverse electrical conduction).

transversale. On écrit la conductivité isotrope transverse sous la forme du tenseur d'ordre deux suivant :

$$
\underline{\boldsymbol{\sigma}}^{(i)}=\sigma_{T}^{(i)}\left(\underline{\boldsymbol{e}}_{r} \otimes \underline{\boldsymbol{e}}_{r}+\underline{\boldsymbol{e}}_{\theta} \otimes \underline{\boldsymbol{e}}_{\theta}\right)+\sigma_{L}^{(i)} \underline{\boldsymbol{e}}_{1} \otimes \underline{\boldsymbol{e}}_{1},
$$

Tableau 1. Dimensions théoriques $\delta^{(i)}$ dans le cas des diamètres $d=0.506$ et $2.064 \mathrm{~mm}$, avec les conductivités locales $\sigma^{(i)}\left(\mu \Omega^{-1} \mathrm{~cm}^{-1}\right)$ à $293 \mathrm{~K}$ et la fraction volumique $f^{(i)}$ de chaque phase $i$.

Table 1. Theoretical dimensions $\delta^{(i)}$ in the case of diameters $d=0.506$ and $2.064 \mathrm{~mm}$, with the corresponding local conductivity $\sigma^{(i)}\left(\mu \Omega^{-1} \mathrm{~cm}^{-1}\right)$ at $293 \mathrm{~K}$ and volume fraction $f^{(i)}$ of each phase $i$.

\begin{tabular}{cccccc}
\hline \multirow{2}{*}{ phase $i$} & \multirow{2}{*}{$f^{(i)}$} & \multicolumn{2}{c}{$d=0.506 \mathrm{~mm}$} & \multicolumn{2}{c}{$d=2.064 \mathrm{~mm}$} \\
& & $\delta^{(i)}$ & $\sigma^{(i)}$ & $\delta^{(i)}$ & $\sigma^{(i)}$ \\
\hline $\mathrm{Nb}-\mathrm{t}$ & $23.8 \%$ & $83 \mathrm{~nm}$ & 0.057 & $338 \mathrm{~nm}$ & 0.060 \\
$\mathrm{Cu}-\mathrm{f}$ & $4.5 \%$ & $110 \mathrm{~nm}$ & 0.424 & $450 \mathrm{~nm}$ & 0.533 \\
$\mathrm{Cu}-0$ & $16.5 \%$ & $71 \mathrm{~nm}$ & 0.372 & $289 \mathrm{~nm}$ & 0.513 \\
$\mathrm{Cu}-1$ & $13.7 \%$ & $492 \mathrm{~nm}$ & 0.536 & $2006 \mathrm{~nm}$ & 0.560 \\
$\mathrm{Cu}-2$ & $18.0 \%$ & $5.58 \mu \mathrm{m}$ & 0.565 & $22.8 \mu \mathrm{m}$ & 0.568 \\
$\mathrm{Cu}-3$ & $23.4 \%$ & $31.6 \mu \mathrm{m}$ & 0.568 & $129 \mu \mathrm{m}$ & 0.568 \\
$\mathrm{Cu}$ massif & $\infty$ & 0.568 & $\infty$ & 0.568 \\
$\mathrm{Nb}$ massif & $\infty$ & 0.060 & $\infty$ & 0.060 \\
\hline
\end{tabular}

avec $\otimes$ le produit tensoriel. La loi de comportement dans la phase $i$ obéit à la loi d'Ohm :

$$
\underline{\boldsymbol{j}}^{(i)}=-\boldsymbol{\sigma}^{(i)} \cdot \nabla \Phi^{(i)}=\boldsymbol{\sigma}^{(i)} \underline{\boldsymbol{E}}^{(i)}
$$

où $\Phi, \underline{j}$ et $\underline{\boldsymbol{E}}^{(i)}$ représentent respectivement le potentiel électrique $(\mathrm{V})$, la densité de courant électrique $\left(\mathrm{A} \mathrm{m}^{-2}\right)$ 
et le champ électrique $\left(\mathrm{V} \mathrm{m}^{-1}\right)$. On fait l'hypothèse d'un régime quasi-statique, d'où :

$$
\operatorname{div}(\underline{\boldsymbol{j}})=0 .
$$

À partir des équations (1) et (2), on obtient l'équation d'équilibre de $\Phi^{(i)}$ dans la phase $i$ :

$$
-\sigma_{T}^{(i)} \frac{\partial^{2} \Phi^{(i)}}{\partial^{2} r}-\frac{\sigma_{T}^{(i)}}{r}\left[\frac{\partial^{2} \Phi^{(i)}}{r \partial \theta^{2}}+\frac{\partial \Phi^{(i)}}{\partial r}\right]-\sigma_{L}^{(i)} \frac{\partial^{2} \Phi^{(i)}}{\partial x_{1}^{2}}=0 .
$$

Nous nous intéressons dans un premier temps à la conduction électrique transversale. Pour cela, un champ électrique à distance est appliqué sur la surface de l'échantillon $\left(r=R_{n+1}\right.$ avec $\left.r \rightarrow \infty\right)$ selon la direction transversale $x_{2}$ (voir Fig. 2)

$$
\left.\underline{\boldsymbol{E}}\right|_{r \rightarrow \infty}=E_{0} \underline{\boldsymbol{e}}_{2} .
$$

Le potentiel électrique solution dans la phase $i$ qui satisfait à la fois l'équation d'équilibre (3) et la condition aux limites (4) est proposé sous la forme :

$$
\Phi^{(i)}(r, \theta)=\left(A_{i} r+B_{i} \frac{R_{i-1}^{2}}{r}\right) \cos \theta
$$

où $A_{i}$ and $B_{i}$ sont les constantes à déterminer. Afin d'éviter un problème de singularité en $r=0$, la valeur $B_{1}=0$ est choisie, sans perte de généralité. La densité de courant électrique correspondante selon la direction normale $\underline{\boldsymbol{r}}$ est alors donnée par :

$$
j_{r}^{(i)}(r, \theta)=-\sigma_{T}^{(i)}\left(A_{i}-B_{i} \frac{R_{i-1}^{2}}{r^{2}}\right) \cos \theta .
$$

Les interfaces $\left(r=R_{i}\right)$ étant supposées parfaites, le potentiel électrique $\Phi$ et la densité de courant normale $j_{r}$ doivent vérifier la condition de continuité à l'interface :

$$
\left\{\begin{array}{l}
\Phi^{(i)}\left(R_{i}, \theta\right)=\Phi^{(i+1)}\left(R_{i}, \theta\right) \\
j_{r}^{(i)}\left(R_{i}, \theta\right)=j_{r}^{(i+1)}\left(R_{i}, \theta\right) .
\end{array}\right.
$$

Pour déterminer la valeur des constantes $A_{i}$ et $B_{i}$ du problème cylindrique n-phase, Hervé et Zaoui [17] ont proposé d'utiliser les "matrices de transfert" $\boldsymbol{J}_{\boldsymbol{i}}$ sur les interfaces $\left(r=R_{i}\right)$. La continuité en $r=R_{i}$ devient alors :

$$
\boldsymbol{J}_{\boldsymbol{i}}\left(R_{i}\right) \cdot \boldsymbol{W}_{\boldsymbol{i}}=\boldsymbol{J}_{\boldsymbol{i}+\mathbf{1}}\left(R_{i}\right) \cdot \boldsymbol{W}_{\boldsymbol{i}+\mathbf{1}}
$$

avec $\boldsymbol{W}_{\boldsymbol{i}}$ désignant $\left(A_{i} B_{i}\right)^{T}$, et $\boldsymbol{J}_{\boldsymbol{i}}$ est la matrice suivante :

$$
\boldsymbol{J}_{\boldsymbol{i}}(r)=\left(\begin{array}{cc}
r & \frac{R_{i-1}^{2}}{r} \\
-\sigma_{T}^{(i)} & \sigma_{T}^{(i)} \frac{\stackrel{R_{i-1}^{2}}{r^{2}}}{}
\end{array}\right) .
$$

Définissons les matrices $\boldsymbol{N}$ et $\boldsymbol{Q}$ à partir des matrices $J_{i}:$

$$
\boldsymbol{N}^{(i)}=\boldsymbol{J}_{\boldsymbol{i + 1}}^{-\mathbf{1}}\left(R_{i}\right) \cdot \boldsymbol{J}_{\boldsymbol{i}}\left(R_{i}\right) \text { et } \boldsymbol{Q}^{(\boldsymbol{k})}=\prod_{i=k}^{1} \boldsymbol{N}^{(i)} .
$$

L'équation (8) est réécrite sous la forme :

$$
W_{i+1}=N^{(i)} \cdot W_{i}, \quad W_{k+1}=Q^{(k)} W_{1} .
$$

Tous les coefficients $A_{k}, B_{k}(k \in[1, n])$ peuvent alors être calculés :

$$
\left\{\begin{array}{l}
A_{k}=\frac{Q_{11}^{(k-1)}}{Q_{11}^{(n)}} A_{n+1} \\
B_{k}=\frac{Q_{21}^{(k-1)}}{Q_{11}^{(n)}} A_{n+1}
\end{array}\right.
$$

avec $A_{n+1}=-E_{0}$. La densité de courant électrique $\underline{\boldsymbol{j}}^{(i)}$ et le potentiel $\Phi^{(i)}$ sont maintenant déterminés dans toutes les phases $i(i \in[1, n])$.

Dans le cas de la conduction électrique longitudinale, l'équation d'équilibre (3) se met sous la forme suivante dans chaque phase :

$$
\Phi^{(i)}=-E_{0} x_{1}
$$

pour des conditions aux limites homogènes au contour : $\left.\underline{j}\right|_{r \rightarrow \infty} \underline{e}_{r}=0$ sur la surface latérale du cylindre et $\bar{\Phi}=-E_{0} x_{1}$ sur les deux sections extrêmes, avec $E_{0}$ un champ électrique constant. On obtient alors une densité de courant homogène par phase, contrairement au cas transversal.

\subsubsection{Schéma autocohérent généralisé}

Afin de déterminer la conductivité effective $\widetilde{\boldsymbol{\sigma}}$ du composite $\mathrm{Cu}-\mathrm{Nb}$, le problème cylindrique $n$-phase résolu précédemment est utilisé avec une phase $(n+1)$ inconnue représentant le MHE, et une condition d'auto-cohérence (qui peut être une condition énergétique telle que celle utilisée par Christensen et Lo [23] ou une condition portant sur les moyennes de champs [24], ces deux conditions étant équivalentes). Cette démarche a été suivie par Joannes et Hervé-Luanco [19] et conduit, dans le cas de la conductivité transversale, à $B_{n+1}=0$ et, par voie de conséquence à $Q_{12}^{(n)}=0$, entrainant :

$$
\tilde{\sigma}_{T}=\sigma_{T}^{(n+1)}=\frac{\sigma_{T}^{(n)}\left[R_{n}^{2} Q_{11}^{(n-1)}-R_{n-1}^{2} Q_{21}^{(n-1)}\right]}{R_{n}^{2} Q_{11}^{(n-1)}+R_{n-1}^{2} Q_{21}^{(n-1)}} .
$$

Dans le cas de la conductivité longitudinale, la condition d'auto-cohérence conduit à :

$$
\tilde{\sigma}_{L}=\sum f^{(i)} \sigma_{L}^{(i)}
$$

avec $f^{(i)}$ la fraction volumique de la phase $i$. On remarquera que cette dernière expression n'est autre que la loi des mélanges sur les conductivités. Il s'agit en fait de la solution exacte pour toutes les microstructures formées de fils parallèles, quel que soit l'assemblage de ces fils. 
(a)

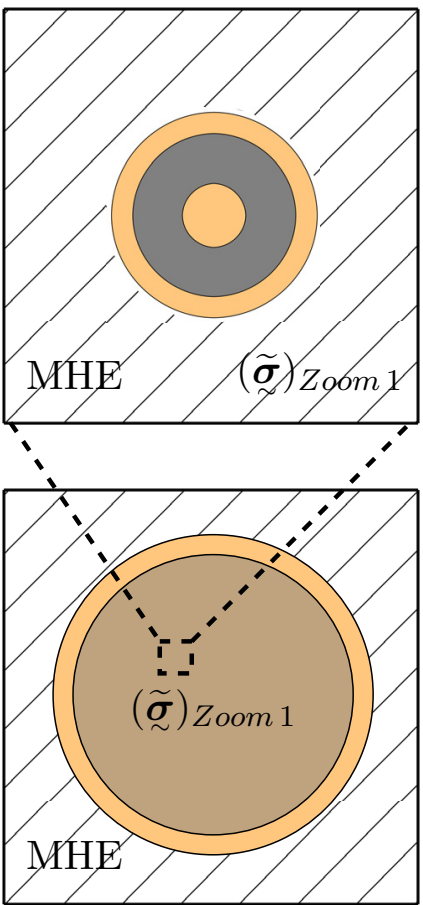

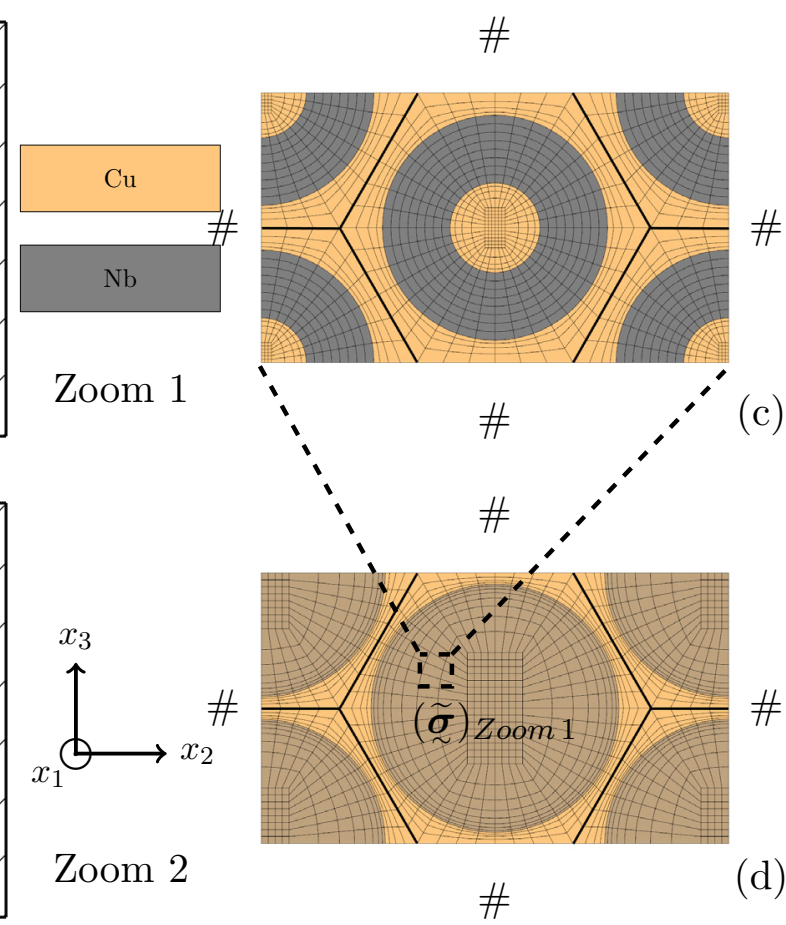

Fig. 3. Modélisation multi-échelle de la conductivité effective du Zoom 1 (a et c) au Zoom 2 (b et d). (a, b) : processus itératif par modèle Auto-Cohérent Généralisé (ACG); (c, d) : Homogénéisation Périodique (HP) avec les maillages de la méthode des éléments finis $(\mathrm{MEF})$.

Fig. 3. Multi-scale modeling of the effective conductivity from the Zoom 1 ( $a$ and $c$ ) to the Zoom 2 ( $b$ and $d$ ). $(a, b)$ : the iterative process by the Generalized Self-Consistent model (GSC); (c, d): Periodic Homogenization (PH) with the finite element method (FEM).

\subsection{Homogénéisation périodique}

Le modèle analytique ACG présenté ci-dessus suppose que les fibres longues sont distribuées aléatoirement (Fig. 3a). Afin de tenir compte de la distribution quasi-périodique observée expérimentalement (Fig. 1), résultant du processus d'élaboration, et d'étudier l'effet de cette distribution particulière, le problème périodique a été résolu. Pour cela, nous avons considéré une cellule élémentaire soumise à des conditions aux limites périodiques, et le problème (que nous appelons HP pour Homogénéisation Périodique) a été résolu par la méthode des éléments finis [20-22]. La périodicité de la cellule de base et des conditions aux limites permettent de déterminer la conductivité effective $\widetilde{\sim}$ du MHE correspondant, i.e. infini et périodique.

La cellule de base considérée dans le modèle HP, ainsi que le maillage utilisé (éléments quadratiques à 20 nœuds, c3d20), sont indiqués sur la figure 3c. La cellule de base contient toute l'information morphologique du Volume Élémentaire Représentatif (VER). Elle contient deux fibres longues $(1+4 \times 1 / 4$ fibres $)$ disposées sur un réseau hexagonal, et elle représente la microstructure expérimentale (idéalisée) des nano-composites $\mathrm{Cu}-\mathrm{Nb}$ sur le Zoom 1. Ces calculs HP sont basés sur des conditions aux limites et des microstructures périodiques. Or la microstructure réelle ne l'est pas, notamment car chaque Zoom ne contient finalement qu'un nombre limité de motifs élémentaires (85). Nous avons vérifié à l'aide de calculs EF sur de plus grandes structures avec conditions aux limites non périodiques que cette hypothèse de périodicité ne modifiait guère les résultats.

Sous conditions aux limites périodiques, le potentiel $\Phi$ dans le volume $V$ s'écrit de la manière suivante :

$$
\Phi=\langle\nabla \Phi\rangle \cdot \underline{x}+t, \quad \forall \underline{x} \in V
$$

où $\langle\underline{\boldsymbol{a}}\rangle$ désigne la moyenne volumique :

$$
\langle\underline{\boldsymbol{a}}\rangle=\frac{1}{V} \int_{V} \underline{\boldsymbol{a}} \mathrm{d} V .
$$

Dans l'équation (15), $t$ est la fluctuation périodique, i.e. deux points homologues sur des faces opposées de $V$ ont la même valeur de $t$. En outre, le scalaire $\underline{\boldsymbol{j}} \cdot \underline{\boldsymbol{n}}$ prend des valeurs opposées en deux points homologues ( $\underline{\boldsymbol{n}}$ étant le vecteur normal de $\partial V$ en $\underline{\boldsymbol{x}}$ ).

Afin de déterminer les deux composantes de la conductivité électrique, on applique successivement, comme conditions aux limites sur $V$, le champ électrique $\underline{\boldsymbol{E}}$ suivant : $\langle\nabla \Phi\rangle=E_{0} \boldsymbol{e}_{1}$ pour la conductivité longitudinale, et $\langle\nabla \Phi\rangle=E_{0} \underline{\underline{\boldsymbol{e}_{2}}}$ (ou $E_{0} \underline{\underline{\boldsymbol{e}_{3}}}$ ) pour la conductivité transversale. Puis, la densité de courant moyenne $\langle\boldsymbol{j}\rangle$ est déterminée par homogénéisation numérique, afin d'obtenir la conductivité effective HP à partir de la condition suivante :

$$
\langle\nabla \Phi\rangle=\tilde{\sim} \cdot\langle\underline{j}\rangle .
$$




\section{Application à la conductivité effective des nano-composites $\mathrm{Cu}-\mathrm{Nb}$}

\subsection{Comportement local}

Les phases $\mathrm{Cu}$ et $\mathrm{Nb}$ évoquées ci-dessus sont en réalité elles-mêmes constituées de grains nanométriques (polycristaux). Cependant, ces deux métaux étant de structure cristalline cubique, la conductivité à l'échelle des grains est isotrope (Sivardière [25]), et donc, du point de vue de la conductivité, les phases polycristallines de $\mathrm{Cu}$ et $\mathrm{Nb}$ peuvent être considérées comme homogènes, les propriétés effectives de ces polycristaux étant identiques à celle des grains constitutifs. Ainsi, les conductivités longitudinale $\sigma_{L}^{(i)}$ et transversale $\sigma_{T}^{(i)}$ sont égales, pour $\mathrm{Cu}$ et pour $\mathrm{Nb}$.

En outre, Thilly [5] a montré que la température $T$, la densité de dislocations $\beta$ et l'effet de taille (largeur des canaux) peuvent avoir un effet significatif sur la conductivité électrique locale des nano-composites $\mathrm{Cu}-\mathrm{Nb}$. La conductivité d'échantillons massifs de $\mathrm{Cu}$ et de $\mathrm{Nb}$ a été mesurée, en fonction de $T$ et $\beta$, dans [5]. Il a ainsi été montré que, à température ambiante $(293 \mathrm{~K})$, dans le cas des conducteurs fortement écrouis de diamètre $d \in(0.5,2.5) \mathrm{mm}$, la densité de dislocations $\beta$ influence peu la conductivité. Cela correspond au cas de fortes densités de dislocation, probablement saturantes, estimées à $\beta \sim 10^{16} \mathrm{~m}^{-2}$ dans les canaux larges, et de l'ordre de $10^{12}$ à $10^{13} \mathrm{~m}^{-2}$ dans les canaux fins. Pour ces échantillons massifs, les valeurs de conductivité sont, à $293 \mathrm{~K}: \sigma_{\text {massif }}=0.568 \mu \Omega^{-1} \mathrm{~cm}^{-1}$ pour $\mathrm{Cu}$, et $0.060 \mu \Omega^{-1} \mathrm{~cm}^{-1}$ pour $\mathrm{Nb}$ (voir aussi le Tab. 1). Les composites $\mathrm{Cu}-\mathrm{Nb}$ étudiés ici présentent des densités de dislocations similaires, associées aux grandes déformations à froid, et donc l'effet de $\beta$ ne sera pas pris en compte dans la suite.

Concernant l'effet de taille, nous utilisons l'expression issue de Ashcroft et Mermin [26] et Thilly [5] qui relie la conductivité du matériau au libre parcours moyen $\ell$ des électrons :

$$
\begin{aligned}
& \ell_{\mathrm{Cu}}(T)=66 \sigma_{\text {massif-Cu}}(T) \\
& \ell_{\mathrm{Nb}}(T)=87 \sigma_{\text {massif-Nb }}(T)
\end{aligned}
$$

(avec $\sigma_{\text {massif }}$ en $\mu \Omega^{-1} \mathrm{~cm}^{-1}$ et $\ell$ en $\mathrm{nm}$ ). Ici, $\sigma_{\text {massif }}$ et $\ell$ dépendent de la température, et le libre parcours moyen $\ell$ des électrons est défini comme la distance moyenne que ces derniers peuvent parcourir entre deux collisions. Initialement, Dingle [27] a étudié les effets de taille dans la diffusion des électrons. Il a modélisé la conductivité d'un fil fin, infiniment long, et de diamètre $\delta$, et s'est donc intéressé à la façon dont la conductivité longitudinale est affectée par les dimensions transversales du fil, en raison de la diffusion des électrons aux surfaces/interfaces. Deux cas extrêmes, $\delta \gg \ell$ et $\delta \ll \ell$, ont été considérés. En modifiant le modèle de Dingle [27] and Thilly [5] obtient les équations suivantes en fonction des rapports $\delta / \ell$ :

$$
\frac{\sigma}{\sigma_{\text {massif }}}=\frac{\delta}{\ell}-\frac{3}{8}\left(\frac{\delta}{\ell}\right)^{2}\left[\ln \left(\frac{\ell}{\delta}\right)+1.059\right] \text { pour } \delta<0.467 \ell,
$$

$$
\begin{aligned}
& \frac{\sigma}{\sigma_{\text {massif }}}=\left(1+\frac{\ell}{\delta}\right)^{-1} \quad \text { pour } \delta \in[0.467 \ell, 3 \ell[, \\
& \frac{\sigma}{\sigma_{\text {massif }}}=1-\frac{3}{4} \frac{\ell}{\delta} \quad \text { pour } \delta>3 \ell .
\end{aligned}
$$

Le choix de l'une de ces trois équations pour le calcul de la conductivité locale $\sigma^{(i)}$ d'une phase $i$ se fait en fonction du rapport $\delta^{(i)} / \ell$, où $\delta^{(i)}$ est la dimension théorique des différentes phases $i$ des conducteurs $\mathrm{Cu}-\mathrm{Nb}$ (Fig. 4). Les valeurs de $\delta^{(i)}$ peuvent être estimées en supposant que toutes les phases forment des cylindres ou des tubes parfaits et concentriques déformés de manière homothétique lors de l'étirage du composite [7]. Le diamètre extérieur $d \mathrm{du}$ fil considéré, ainsi que la fraction volumique $f^{(i)}$ des composants doivent être pris en compte. La fraction volumique, qui n'est pas modifiée par l'étape d'étirage, est déterminée à partir des dimensions initiales des tubes et fils de $\mathrm{Cu}$ et $\mathrm{Nb}$ introduits dans le procédé. Nous désignons par $e_{\mathrm{Nb}-\mathrm{t}}$ l'épaisseur des tubes de niobium, $d_{\mathrm{Cu}-\mathrm{f}}$ le diamètre des filaments de cuivre, et $d_{\mathrm{Cu}-\mathrm{i}}$ la largeur des canaux de la matrice multi-échelle de cuivre, avec $i=0,1,2,3$. Ainsi, $\delta^{(i)}=d_{\mathrm{Cu}-0}$ pour la phase $\mathrm{Cu}-0$, $\delta^{(i)}=d_{\mathrm{Cu}-\mathrm{f}}$ pour $\mathrm{Cu}-\mathrm{f}$, et $\delta^{(i)}=e_{\mathrm{Nb}-\mathrm{t}}$ pour les tubes de Nb. Ces valeurs sont reportées dans le Tableau 1. Dans ce travail, nous considérons une série des conducteurs nanocomposites $\mathrm{Cu}-\mathrm{Nb}$ contenant $85^{3}$ motifs élémentaires et étirés aux diamètres $d=0.506,0.592,0.640,0.692,0.748$, $0.875,1.023,1.511,1.634$ et $2.064 \mathrm{~mm}$ [7]. Le Tableau 1 indique la dimension $\delta^{(i)}$ dans la phase $i$, la fraction volumique $f^{(i)}$ et les conductivités locales $\sigma^{(i)}$ à $293 \mathrm{~K}$ correspondantes, calculées par les equations (18) et (19), pour le plus grand $(d=2.064 \mathrm{~mm})$ et le plus petit $(d=0.506 \mathrm{~mm})$ diamètre.

\subsection{Homogénéisation multi-échelle}

Une fois la conductivité locale $\sigma^{(i)}$ dans chaque phase $i$ déterminée (Sect. 3.1), le modèle Auto-Cohérent Généralisé (Fig. 3a) ainsi que le modèle Homogénéisation Périodique (Fig. 3c) sont tous les deux appliqués à l'échelle du Zoom 1, en considérant une microstructure de motifs co-cylindriques avec trois couches, $\mathrm{Cu}-\mathrm{f} / \mathrm{Nb}-\mathrm{t} / \mathrm{Cu}-0$. Avec ces deux modèles, nous obtenons des conductivités effectives $\underset{\sim}{\widetilde{\sigma}}$ pratiquement confondues, l'écart relatif entre les deux estimations étant $\sim 0.5 \%$.

À l'échelle du Zoom 2, les fibres longues sont composées de deux couches : (1) les nano-composites $\mathrm{Cu}-\mathrm{Nb}$ qui contiennent 85 motifs parallèles issus du Zoom 1, et (2) la matrice de $\mathrm{Cu}-1$. Similairement au cas du Zoom 1, les approximations de la géométrie de ACG et $\mathrm{HP}$ sont illustrées dans les figures $3 \mathrm{~b}$ et $3 \mathrm{~d}$ : répartition aléatoire pour ACG, et périodique pour HP. Dans le processus itératif, la conductivité de la couche interne du 


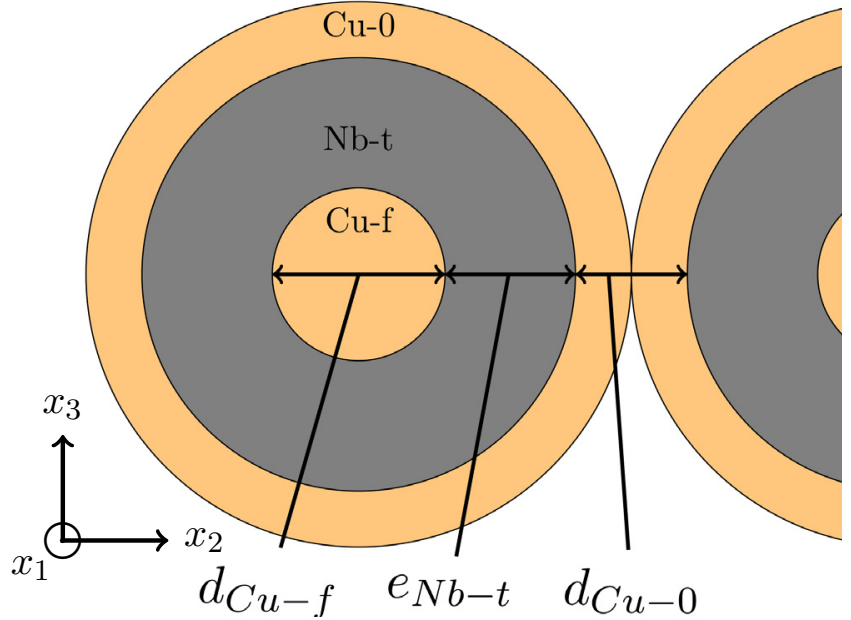

Fig. 4. Largeur théorique des canaux $\delta$ : sur le Zoom 1, $e_{\mathrm{Nb}-\mathrm{t}}$ désigne l'épaisseur des tubes de niobium, $d_{\mathrm{Cu}-\mathrm{f}}$ le diamètre des filaments de cuivre, $d_{C u-0}$ la largeur des canaux de cuivre.

Fig. 4. Theoretical channel width $\delta$ : on the Zoom 1, e $e_{\mathrm{Nb}-\mathrm{t}}$ denotes the thickness of the niobium tubes, $d_{\mathrm{Cu}-\mathrm{f}}$ the diameter of the copper filaments, $d_{\mathrm{Cu}-0}$ copper channel width.

Zoom $2 \quad\left(\boldsymbol{\sigma}^{(1)}\right)$ Zoom 2 est donnée par la conductivité effective obtenue sur le Zoom 1 ( $\underset{\sim}{\tilde{\sigma}})_{\text {Zoom 1. D'autre part, la }}$ conductivité de la deuxième couche du Zoom 2 est donnée par la conductivité locale de $\mathrm{Cu}-1$, i.e. $\left(\boldsymbol{\sigma}^{(2)}\right)_{\text {Zoom } 2}=$ $(\underset{\sim}{\boldsymbol{\sigma}})_{\mathrm{Cu}-1}$. Ainsi, une procédure de calcul multi-échelle est mise en place pour les modèles ACG et HP, comme illustré sur les figures $3 \mathrm{a}-3 \mathrm{c}$ et $3 \mathrm{~b}-3 \mathrm{~d}$. Les conductivités effectives sur le Zoom $2(\underset{\sim}{\widetilde{\sigma}})_{\text {Zoom } 2}$ ainsi obtenues avec les deux modèles sont encore pratiquement confondues. Le même processus itératif est répété jusqu'à échelle macroscopique (Zoom 4), permettant d'estimer les conductivités à toutes les échelles $(\underset{\sim}{\tilde{\boldsymbol{\sigma}}})_{\text {Zoom i }}$ avec $i=2,3,4$ :

$$
\left.\begin{array}{c}
\left({\underset{\sim}{\boldsymbol{\sigma}}}^{(1)}\right)_{\mathrm{Zoom} i}=(\underset{\sim}{\tilde{\boldsymbol{\sigma}}})_{\mathrm{Zoom}(i-1)} \\
\left({\underset{\sim}{\boldsymbol{\sigma}}}^{(2)}\right)_{\mathrm{Zoom} i}=(\underset{\sim}{\boldsymbol{\sigma}})_{\mathrm{Cu}-(i-1)}
\end{array}\right\} \Rightarrow(\underset{\sim}{\tilde{\boldsymbol{\sigma}}})_{\mathrm{Zoom} i} \Rightarrow \ldots(\underset{\sim}{\tilde{\boldsymbol{\sigma}}})_{\text {macro }} .
$$

La figure 5 illustre les résultats sur le Zoom 4 à $293 \mathrm{~K}$ : les traits continus reportent les résultats obtenus avec le modèle analytique ACG et les points ceux du modèle par éléments finis HP. Il est remarquable de constater que les deux conductivités effectives, longitudinale $\widetilde{\sigma}_{L}$ et transversale $\widetilde{\sigma}_{T}$, obtenues avec les deux modèles, sont quasiment confondues, pour tous les diamètres du conducteur $d$. Le faible écart entre ces deux prédictions s'explique par le fait que (i) le contraste entre la conductivité des deux phases reste faible pour $\mathrm{Cu}-\mathrm{Nb}$, et (ii) les deux modèles prennent en compte la présence d'une phase jouant le rôle de matrice. La diminution de la conductivité $\tilde{\sim}$ avec la réduction du diamètre $d$ est due à la réduction des largeurs des canaux $\delta$ associée à l'étirage (effet de taille).

\section{Comparaison expérimentale}

Dubois [7] a déterminé expérimentalement la conductivité longitudinale effective $\widetilde{\sigma}_{L}$ sur la même série de

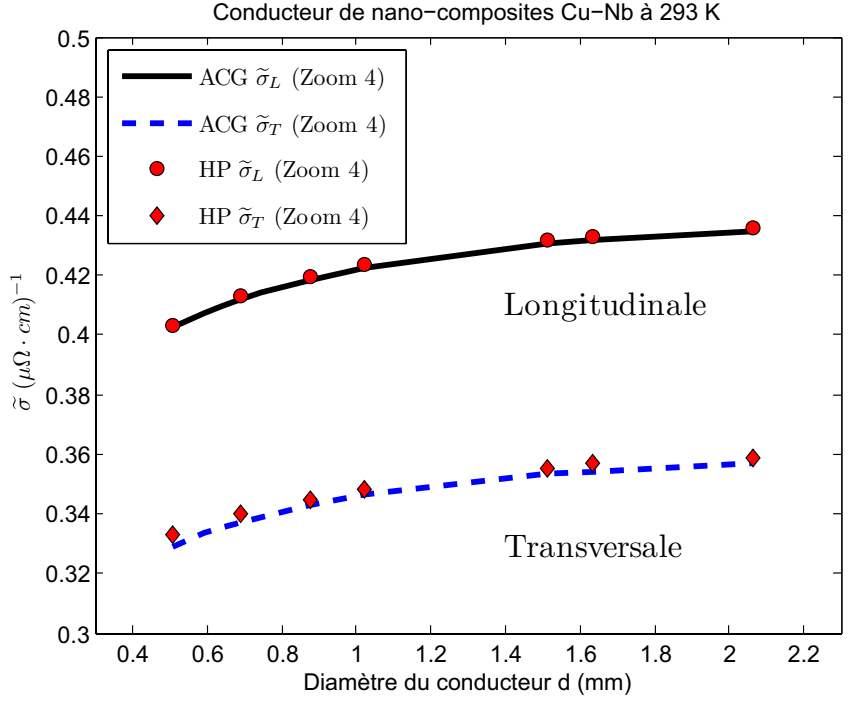

Fig. 5. Conductivités effectives longitudinale et transversale en fonction du diamètre du conducteur, pour les fils de nano-composites $\mathrm{Cu}-\mathrm{Nb}$ sur le Zoom 4 (i.e. à l'échelle macroscopique) à $293 \mathrm{~K}$, obtenues par le modèle Auto-Cohérent Généralisé (ACG, traits continus) et par Homogénéisation Périodique (HP, points).

Fig. 5. Effective longitudinal and transverse conductivities with respect to the conductor diameter, for nano-composite $\mathrm{Cu}-\mathrm{Nb}$ wires on Zoom 4 (i.e. at the macroscopic scale) at $293 \mathrm{~K}$, obtained with the Generalized Self-Consistent model (GSC, continuous lines) and the Periodic Homogenization model (PH, points).

conducteurs nano-composites $\mathrm{Cu}-\mathrm{Nb}$ par la méthode des quatre pointes [28] : un courant continu d'intensité $j$ est injecté entre deux pointes et une mesure de tension est réalisée entre deux autres pointes placées entre les pointes d'injection de courant. La conductivité transversale n'a pas encore pu être mesurée (travail en cours); il s'agit en effet d'une mesure plus délicate, en raison du faible diamètre des fils, mais aussi de sa microstructure composite avec une peau en $\mathrm{Cu}(\mathrm{Cu}-3)$ entourant le fil.

L'incertitude sur la mesure de conductivité est de l'ordre de $5 \%$ pour les échantillons composites dans la direction longitudinale $\widetilde{\sigma}_{L}$ et pour les échantillons massifs $\sigma_{\text {massif }}$. Les résultats des modèles ACG et HP étant directement dépendants de la valeur de la conductivité $\sigma_{\text {massif }}$ de chacune des phases polycristallines $\mathrm{Cu}$ et $\mathrm{Nb}$, l'incertitude de mesure sur $\sigma_{\text {massif }}$ entraîne une incertitude sur les prédictions du modèle, également de l'ordre de $\pm 5 \%$.

La comparaison avec les données expérimentales est illustrée sur la figure 6. Rappelons que la conductivité longitudinale obtenue par le modèle Auto-Cohérent Généralisé (ACG) est ici confondue avec les résultats du modèle périodique (HP); en effet, la conductivité longitudinale, dont la valeur exacte est donnée par la loi des mélanges, ne dépend pas du caractère périodique de la microstructure. La figure 6 montre que les résultats des modèles théoriques sont en très bon accord avec les données expérimentales, pour tous les diamètres considérés. Ce résultat provient d'une prise en compte 


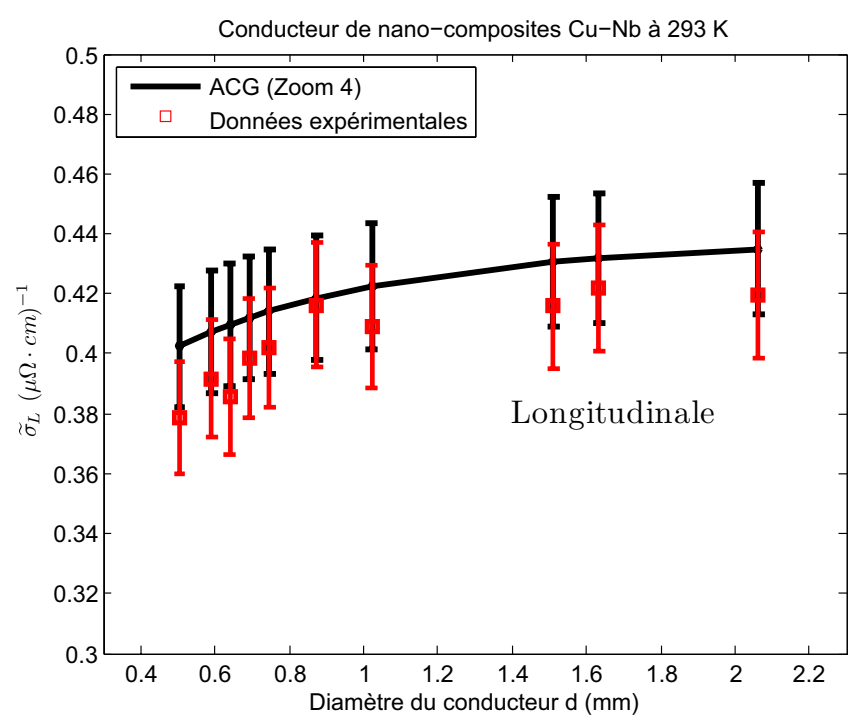

Fig. 6. Conductivité effective longitudinale obtenue avec le modèle ACG (Auto-Cohérent Généralisé, trait continu), et comparée aux données expérimentales (points), en fonction du diamètre du conducteur, pour les fils de nano-composites $\mathrm{Cu}-\mathrm{Nb}$ sur le Zoom 4 (échelle macroscopique) à $293 \mathrm{~K}$. L'incertitude sur la mesure de la conductivité des échantillons massifs $(\sim 5 \%)$ entraîne une incertitude du même ordre sur les résultats du modèle ACG.

Fig. 6. Effective longitudinal conductivity obtained with the GSC model (Generalized Self-Consistent scheme, continuous line), compared with experimental data (points), for various conductor diameters, for nano-composite $\mathrm{Cu}-\mathrm{Nb}$ wires on the Zoom 4 (macroscopic scale) at $293 \mathrm{~K}$. Uncertainties on the measurement of resistivity on bulk specimen ( $\sim 5 \%)$ leads to an uncertainty of a similar magnitude on model results.

pertinente (i) de la conductivité locale et (ii) des transitions des échelles dans les modèles utilisés.

\section{Conclusions et perspectives}

En prenant en compte les effets de taille (ou largeur des canaux $\delta$ ), la température $T$ et la densité de dislocations $\beta$ à l'aide de la loi Dingle modifiée, nous avons déterminé la conductivité locale $\sigma^{(i)}$ dans chaque phase $i$. Par un processus itératif, le caractère multi-échelle est pris en compte dans le modèle analytique ACG et dans les calculs par éléments finis HP. Les conductivités effectives $\widetilde{\sigma}$ obtenues par ACG et HP sont parfaitement confondues dans les directions longitudinale et transversale.

$\mathrm{Au}$ niveau de l'approximation de la géométrie, nous avons considéré une microstructure formée de motifs co-cylindriques répartis aléatoirement par ACG et périodiquement par HP. Les résultats sur ces deux modélisations sont pratiquement confondus sur tous les zooms. Ce résultat suggère que la distribution des fibres longues joue un rôle mineur sur la conductivité effective $\widetilde{\sigma} \mathrm{du}$ conducteur $\mathrm{Cu}-\mathrm{Nb}$, dans la direction transversale. Dans la direction longitudinale, il est rappelé que la solution exacte est indépendante du caractère périodique de la microstructure. Ces résultats justifient pleinement l'emploi du modèle ACG en champs moyens pour l'estimation de la conductivité effective, qui permet un gain de temps de calculs très significatif par rapport aux calculs par Eléments Finis. Les conductivités effectives $\widetilde{\sigma}$ déterminées par ACG et HP sont également en bon accord avec les données expérimentales disponibles dans la direction longitudinale.

Ce travail se poursuit actuellement selon les directions suivantes :

1. La méthode classique des quatre pointes ne permet de mesurer que la conductivité longitudinale. Il reste donc à effectuer les mesures de conductivité tranversale pour une validation complète des modèles. Les comparaisons expérimentales à plusieurs températures $T$ seront aussi importantes pour la validation des modèles.

2. Dans ce travail, nous avons utilisé des valeurs théoriques pour les largeurs des canaux $\delta$. Les observations expérimentales montrent cependant une certaine fluctuation de ces largeurs, probablement associées à des hétérogénéités de déformation lors des étapes de mise en forme. Une évaluation plus fine des largeurs effective est en cours pour affiner les prédictions, puisque la largeur des canaux influence sur la conductivité locale.

3. Basée sur les résultats obtenus en conductivité, une approche similaire est en cours afin d'estimer les propriétés élastiques et plastiques, afin de positionner le comportement modélisé de ces fils $\mathrm{Cu}-\mathrm{Nb}$ sur des cartes d'Ashby, et, à terme, pouvoir en tirer les paramètres microstructuraux importants en vue d'une optimisation du matériau et/ou du procédé d'élaboration.

Remerciements. Les auteurs remercient l'ANR pour le financement du projet METAFORES ANR-12-BS09-0002.

\section{Références}

[1] J. Béard, J. Billette, P. Frings, M. Suleiman, F. Lecouturier, J. Low Temperature Phys. 170 (2013) 442-446

[2] National Research Council, High Magnetic Field Science and Its Application in the United States : Current Status and Future Directions, The National Academies Press, 2013

[3] L. Frydman, J. Magnetic Resonance 242 (2014) 256-264

[4] L. Thilly, F. Lecouturier, Les nanosciences 2 : Nanomatériaux et nanochimie, chapter Applications des nanomatériaux : mécanique : bobines hauts champs, Ed. Belin, coll. Echelles, 2006, pp. 650-658

[5] L. Thilly, Exploration théorique et expérimentale de fils nanocomposites continus présentant des propriétés extrêmes de conductivite électrique et de limite élastique, Ph.D. thesis, Institut National des Sciences Appliquées de Toulouse, 2000

[6] J.B. Dubois, L. Thilly, P.-O. Renault, F. Lecouturier, Adv. Eng. Mater. 14 (2012) 998-1003 
[7] J.B. Dubois, Conducteurs nanocomposites métalliques élaborés par déformation plastique sévère : formation et stabilité thermo-mécanique des nanostructures, propriétés induites, Ph.D. thesis, Université de Poitiers, 2010

[8] K. Spencer, F. Lecouturier, L. Thilly, J.D. Embury, Adv. Eng. Mater. 6 (2004) 290-297

[9] L. Thilly, F. Lecouturier, J. Von Stebut, Acta Materialia 50 (2002) 5049-5065

[10] L. Thilly, M. Véron, O. Ludwig, F. Lecouturier, Mater. Sci. Eng. A 309 (2001) 510-513

[11] V. Vidal, L. Thilly, F. Lecouturier, P.-O. Renault, Scripta Materialia 57 (2007) 245-248

[12] J.B. Dubois, L. Thilly, P.-O. Renault, F. Lecouturier, M. Di Michiel, Acta Materialia 58 (2010) 6504-6512

[13] M.J. Demkowicz, L. Thilly, Acta Materialia 59 (2011) 7744-7756

[14] L. Thilly, P.-O. Renault, S. Van Petegem, S. Brandstetter, B. Schmitt, H. Van Swygenhoven, V. Vidal, F. Lecouturier, Appl. Phys. Lett. 90 (2007) 241907

[15] L. Thilly, S. Van Petegem, P.-O. Renault, F. Lecouturier, V. Vidal, B. Schmitt, H. Van Swygenhoven, Acta Materialia 57 (2009) 3157-3169

[16] Z. Hashin, D. Bagchi, B.W. Rosen, Non-linear behavior of fiber composite laminates, NASA (NASA-CR-1974), 1974

[17] E. Hervé, A. Zaoui, Int. J. Eng. Sci. 31 (1993) 1-10
[18] E. Hervé, A. Zaoui, Int. J. Eng. Sci. 33 (1995) 1419-1433

[19] S. Joannes, E. Hervé-Luanco, Multiscale modeling of transport phenomena for materials with n-layered embedded fibres : an analytical and numerical-based approach, Publication in progress.

[20] J. Besson, G. Cailletaud, J.-L. Chaboche, S. Forest, Nonlinear mechanics of materials, Springer, 2009, Vol. 167

[21] S. Forest, G. Cailletaud, D. Jeulin, F. Feyel, I. Galliet, V. Mounoury, S. Quilici, Mécanique $\mathscr{E}$ industries 3 (2002) 439-456

[22] T. Kanit, S. Forest, I. Galliet, V. Mounoury, D. Jeulin, Int. J. Solids Struct. 40 (2003) 3647-3679

[23] R.M. Christensen, K.H. Lo, J. Mech. Phys. Solids 27 (1979) 315-330

[24] E Hervé, Int. J. Solids Struct. 39 (2002) 1041-1058

[25] J. Sivardière, Symétrie et propriétés physiques - Du principe de Curie aux brisures de symétrie, EDP Sciences, 2008

[26] N.W. Ashcroft, N.D. Mermin, Solid State Physics, Saunders College, 1976

[27] R.B. Dingle, Proc. Roy. Soc. London. Ser. A. Math. Phys. Sci. 201 (1950) 545-560

[28] Commission Electrotechnique Internationale, Méthode de mesure de la résistivité des matériaux métalliques, Technical report, Publication CEI 468, 1974 